\title{
Ethiopian Enset Diseases Diagnosis Model Using Image Processing and Machine Learning Techniques
}

\author{
Kibru Abera Ganore ${ }^{1}$, Getahun Tigistu ${ }^{2}$ \\ ${ }^{1}$ Department of Computer Science, Wachemo University, Addis Ababa, Ethiopia \\ ${ }^{2}$ Faculty of Computing and Software Engineering, Arba Minch University, Arba Minch, Ethiopia
}

Email address:

gettigistu@gmail.com (G. Tigistu)

\section{To cite this article:}

Kibru Abera Ganore, Getahun Tigistu. Ethiopian Enset Diseases Diagnosis Model Using Image Processing and Machine Learning Techniques. International Journal of Intelligent Information Systems. Vol. 9, No. 1, 2020, pp. 1-5. doi: 10.11648/j.ijiis.20200901.11

Received: May 14, 2020; Accepted: May 29, 2020; Published: June 17, 2020

\begin{abstract}
The diagnosis of diseases on the plant is a very important to provide large quantity and good qualitative agricultural products. Enset is an important food crops produced in Southern parts of the Ethiopia with great role in food security. There are several issues and diseases which try to decline the yield with quality. Particularly, diagnosis of potential diseases on Enset is based on traditional ways. The aim of this study is to design a model for Enset diseases diagnosis using Image processing and Multiclass SVM techniques. This study presented a general process model to classify a given Enset leaf image as normal or infected. The strategy of K-fold stratified cross validation was used to enhance generalization of the model. This diagnosis apply K-means clustering, color distribution, shape measurements, Gabor texture extraction and wavelet transform as key approaches for image processing techniques. The researcher selected two Enset leaf diseases viz. Bacterial Wilt and Fusarium Wilt disease and collected 430 Enset leaf images from Areka agricultural research center and some selected areas in SNNPR. For this research work MATLAB version R2017a tool was used as a platform to simulate the real world data. The proposed model demonstrated with four different kernels, and the overall result indicates that the RBF Kernel achieves the highest accuracy as $94.04 \%$ and $92.44 \%$ for bacterial wilt and fusarium wilt respectively. Therefore, an efficient practice of IT based solution in this domain will increases productivity and quality of Enset products.
\end{abstract}

Keywords: Multiclass SVM, Kernels, Enset Disease, K-means Clustering, Image Processing

\section{Introduction}

Food security is a challenge in many developing countries like Ethiopia. Nations in our country are still struggling to make use of available resources so as to combat hunger. Around $80 \%$ to $85 \%$ of people in Ethiopia are dependent on agriculture; among these more than $20 \%$ of them depend on Enset crop production in the country. The government committed $15 \%$ to $17 \%$ of expenditures to the sector; it covers $41 \%$ of gross domestic product (GDP), over $90 \%$ of export value and directly supports $80 \%$ of the populations' livelihoods [1]. It is also the sector that is given an overriding focus in the government's plan for growth of the economy as a whole.

Enset crop is related to and resembles the banana plant which is an indigenous plant classified under the monocarpic genus Enset and family Musaceae. This is commonly known as false banana, the Ethiopian banana or the Abyssinian banana. Locally the plant is called Enset and botanically, it is named as Ensete ventricosum (Welw.) Cheesman.

Ethiopia is one of the grand producer of Enset in African continent countries. There are several issues and diseases which tries to decline the yield with quality. Particularly, diagnosis of potential diseases on Ethiopian banana is based on traditional ways and due to limited research attention given to Enset crop production. The visual observation of the experts is the main approach that commonly used for detection and identification of such plant diseases. As a matter of facts, visual or manual detections may have defects in terms of accuracy in detection along with lower precision. There is a potential need for technology supported alternative systems to support the manual identification of Enset crop diseases so as to optimize the accuracy for remedial action. If the Enset crop diseases can be minimized then definitely the production will significantly be increased to contribute to the country's economy towards a new boost. Therefore, the 
implementation of IT based solution in the sector will have a paramount importance to facilitate the activities such as economic, social and ecologic development in the country by increasing efficiency in both quality and quantity of Enset crops production, to sustain dependability of customer preferences and to preserve the ecology [2]. In our country few researchers found the promising solutions to different plant diseases diagnosis such as maize, rose flower, coffee and others using computer vision and machine learning techniques $[3,4,5]$. The general field of image processing was started before a year and now it is used in different areas.

The remaining part of this paper is organized as follows. The types of common Enest diseases are discussed in Section II. In Section III, we present the architecture of proposed system. Experimental results are reported in Section IV. Section $\mathrm{V}$ presents the conclusion and discusses of future works.

\section{Enset Diseases}

Several types of diseases are known to affect Enset plants under field conditions. So far a number of fungal, nematode, viral and bacterial diseases were reported to cause damage at different degrees of intensity that was mainly explored by Quimio research finding since 1992. The occurrence, distribution and the incidence level also indicated to vary from one Enset-growing locality to the other. Therefore, the damage inflicted by each disease also varied. Among various diseases, Enset bacterial wilt and Fusarium Wilt is considered as the most dangers one that reduces Enset yield $[6,7,8]$.

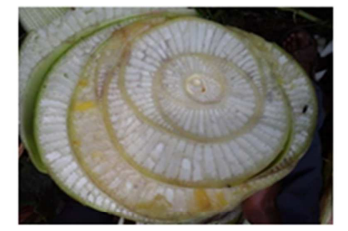

Enset Pseudostem Infected by Bacterial Wilt

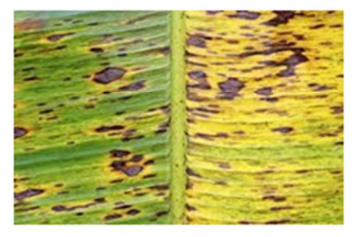

Enset Plant Infected by Fusarium Wilt
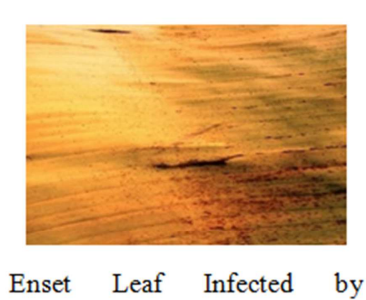
Bacterial Wilt

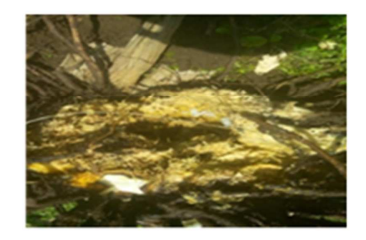

Enset Plant Infected by Fusarium Wilt
Figure 1. Picture of the Infected Enset Plant.

\section{Architecture of Proposed System}

In this work, disease identification was done by using characteristics of Enset plant. The feature of normal and diseased Enset image features was extracted to train kernel support vector machine. As a result a defined Enset image feature repositories was created. Figure 2 shows the architecture of the proposed system

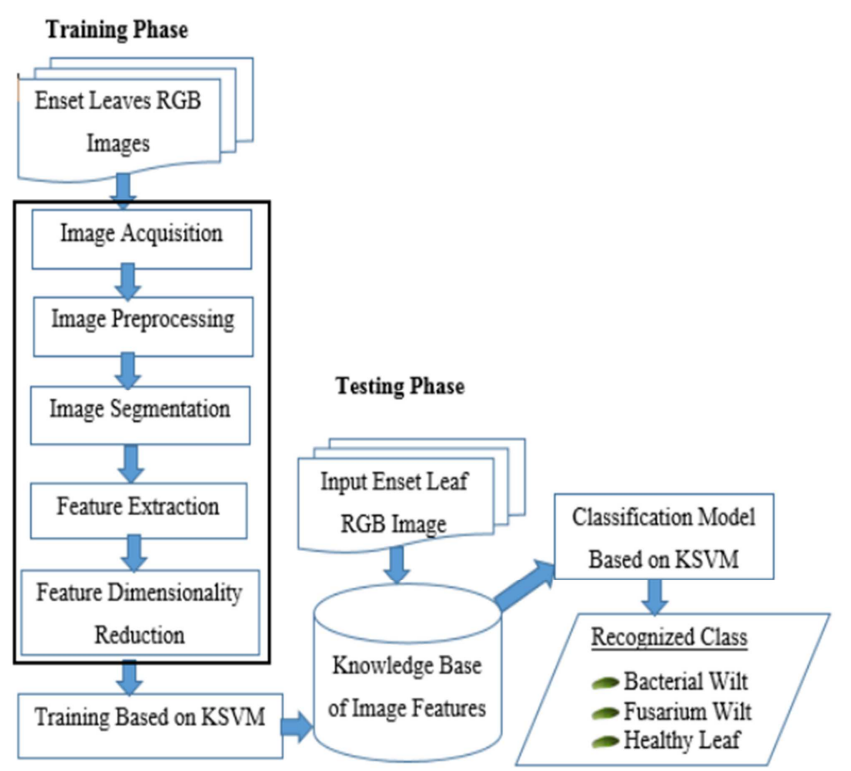

Figure 2. Architecture of the Proposed System.

\section{A. Image Pre-Processing}

The main purpose of this step is to improve the quality of Enset leaf image by removing unrelated and extra parts in the background of the image for further processing. Different pre-processing techniques is applied to the image based on the improvement needed. It may include removing noises, adjusting the intensity or brightness, adding edge detectors etc., based on the desired features for the classification [9, 10, 11].

B. Image Segmentation

Image segmentation is the third image processing step in our proposed model. The segmented plant leaf images are clustered into different sectors using Otsu classifier and kmeans clustering algorithm in order to find the region of interest from the leaf background. Before clustering the images, the input RGB image color model is transformed into $\mathrm{L}^{*} \mathrm{a} * \mathrm{~b}$ color model. The advent of $\mathrm{L} * \mathrm{a} * \mathrm{~b}$ color model is to easily cluster the segmented images in to two spaces [12, 13].

\section{Feature Extraction}

From the image that are preprocessed and segmented three different features that is shape, color, and texture features are extracted. To evaluate the performance of our systems we have used those three different features in different ways. First we tried it independently, then we use the combination of the two and finally we used the three features together.

In the proposed system selection of feature is necessary for classification which is a main problem and challenging issues. So, after the implementation of feature selection algorithm we selected $1 * 129$ sets of features for a single image which are helpful and necessary for computing the classification. Typically, we have extracted $1 * 38$ color feature vectors $(1 * 6$ vector containing the first 2 color moments from each $\mathrm{R}, \mathrm{G}$, $\mathrm{B}$ channel and $1 * 32$ feature vector extracted from HSV color 
space), $1 * 3$ shape measurements and $1 * 88$ feature vector extracted from Gabor texture. All of those features are used to determine whether the Enset plant is infected by the disease and to classify the disease category [14],

i. Color feature

Color features are extracted using color moments and HSV color space. From the HSV color space $1 * 32$ vector features are extracted. Only the first order and second order color moments like mean and standard deviation have been used as the other color feature extraction and extracted $1 * 6$ different feature vectors.

ii. Texture feature

In order to extract a texture feature of the Enset leaf image Gabor texture feature extraction technique is used. From the different Gabor feature extraction technique a discrete Gabor wavelet transformation is used. Gabor wavelet filters spanning four scales: $0.05,0.1,0.2,0.4$ and six orientations: $\Theta 0=0, \Theta n+1=\Theta n+6 / \pi$ are applied to the image. The mean and standard deviation of the Gabor wavelet coefficients are used to form the feature vector. In addition we build a wavelet feature vector using mean squared energy and mean amplitude to generate the wavelet coefficient. Finally, by using Gabor texture feature extraction technique $1 * 88$ feature vectors for a single image were extracted $[14,15]$

Mean Squared Energy $=\sum_{i=1}^{k} \cdot \sum_{j=1}^{k}=A_{i j}^{2} \ldots \ldots \ldots 1$

Mean Amplitude $=\mu_{c} A_{i j} \ldots \ldots \ldots . . .2$

iii. Shape feature

Morphological feature of Enset leaf is the other important feature characterized by external representation of shape, width and height of the given image. A total of three different morphological features that is regional descriptors of minor axis, major axis and circularity of the Enset image pixels were measured and used as a shape features [15].

Table 1. Summery of Features.

\begin{tabular}{|c|c|c|}
\hline Features & Description & Dimensions \\
\hline Color histogram & HSV space is chosen, each $\mathrm{H}, \mathrm{S}, \mathrm{V}$ component is uniformly quantized into 8,2 and 2 bins respectively & 32 \\
\hline Shape features & Regional descriptors of minor axis, major axis and circularity of the Enset image pixels were measured & 3 \\
\hline Color moments & $\begin{array}{l}\text { The first two moments (mean and standard deviation) from the input } R, G, B \text { color channels are extracted } \\
\text { Gabor wavelet filters spanning four scales: } 0.05,0.1,0.2,0.4 \text { and six orientations: } \Theta_{0}=0, \Theta_{n+1}=\Theta_{n}+6 / \pi \text { are }\end{array}$ & 6 \\
\hline Gabor wavelet & $\begin{array}{l}\text { applied to the image. The mean and standard deviation of the Gabor wavelet coefficients are used to form the } \\
\text { feature vector }\end{array}$ & 48 \\
\hline Wavelet moment & $\begin{array}{l}\text { Applying the wavelet transform to the image with a 3-40 level decomposition, the mean } \& \text { the standard } \\
\text { deviation of the transform coefficients are used to form the feature vector }\end{array}$ & 40 \\
\hline
\end{tabular}

In summary, the Enset disease diagnosis model will produce the mean value of six principal feature databases and concatenate the features to form 129-dimensional feature vectors as it is shown in table 1 .

\section{Training}

In this section, the affected images of the two Enset leaf diseases and one healthy leaf were collected from primary and secondary sources and labelled under their diseases category. Then the features of the labelled images are extracted using image analysis as described in the previous sections. This process finally generates a knowledge base which contains a class of Enset leaf image, three prime feature descriptors of the respective image and their respective target class which are the primary inputs for any decision making process. Having these labelled data, we used a supervised machine learning method to identify the class of the image and clearly stated the number of inputs and outputs [16].

Classification of Enset Leaf disease

In order to classify enset disease a multiclass support vector machine was used. The knowledge base that are created during the training phase is used by the multiclass support vector machine to classify the disease in its category. For the three different class of disease category, different labels are used as it is shown below [16, 17].

Classes

$$
\left[\begin{array}{c}
\text { Bacterial Wilt } \\
\text { Fusarium Wilt } \\
\text { Healthy }
\end{array}\right] \rightarrow\left[\begin{array}{c}
\text { Mean }_{2}\langle H|S| V\rangle \text { histogram } \\
\text { Mean }_{2}\langle R|G| B\rangle \text { moment }\left(\begin{array}{c}
\text { mean } \\
\text { Std }
\end{array}\right) \\
\text { Mean } \left._{2}\langle\text { RMinor Axis }| \text { GMajor Axis } \mid \text { BMajor Axis }\right\rangle \\
\text { Mean }_{2}\langle\text { Mean square energy }\rangle \\
\text { Mean }_{2}\langle\text { Mean Amplitude }\rangle \\
\text { Mean }_{2}\langle\text { Wavelet Momente }\rangle
\end{array}\right]=\left[\begin{array}{c}
00001 \\
00010 \\
00011
\end{array}\right]
$$

Figure 3. Vector Representation of Input and Output Features.

\section{Experiment}

A. Dataset Partitioning

The images of the dataset are collected from different parts of southern Ethiopia. Those images are collected by using a digital camera and some of them are collected from secondary sources like research centers which collects pictures for visual inspection of Enset disease. As it is 
mentioned in the above section, for the experiment two diseases and one normal of Enset were identified. From all those disease category a total of 460 Enset leaf images are collected from which 368 is used for training and 92 images are used for testing.

B. Test Result

For the experimentation a total of fifteen experimental setups which is color, texture morphological and a combination of each and a multiclass Kernel support vector machine model: Linear Kernel, polynomial Kernel, RBF kernel and Quadratic Kernel was used.

From the total dataset $20 \%$ is used for testing and the distribution of each disease category is shown in table 2 .

Table 2. Testing Dataset of Enset Leaf Image.

\begin{tabular}{lll}
\hline Class Label number & Type of disease & Number of RGB image \\
\hline 1 & Bacterial Wilt & 30 \\
2 & Fusarium Wilt & 31 \\
3 & Normal image & 32 \\
Total & & 92 \\
\hline
\end{tabular}

Different results were found by using different multiclass support vector machine classifiers. In order to measure the performance of the classifiers K-fold (in this case $\mathrm{k}$ is 10) cross validation method was used. The detail classification result is shown in figure 4. From the figure it is shown that the RBF kernel classifier has classified the given enset image in its correct class category by $94.04 \%$ and $92.44 \%$ respectively.

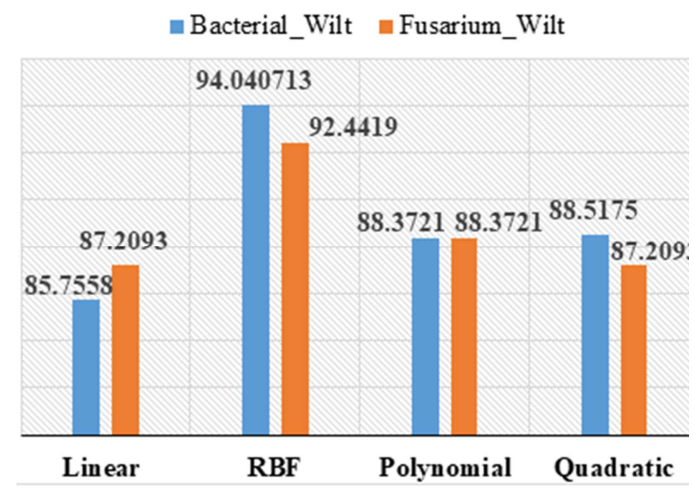

Figure 4. Classification Results of Enset Leaf Diseases.

\section{Conclusion and Future Work}

We designed a system which can detect Enset disease automatically and this will help the farmers to detect the diseases in its early stage and to take relevant action. This can help the farmers to produce a good quality and quantity Enset product. If there is a shortage of experts in the area of those disease identification the system is going to be a replacement for the experts. Better results is achieved if the system is used by large number of dataset. It is a good option to use other machine learning techniques like Artificial Neural network and the hybrids of the other learning algorithms.

\section{References}

[1] MoFED "Macro-economic development in Ethiopia." Addis Ababa, 2015.

[2] A. Ayale and M. Yeshitila. "The response of enset (enset ventricosum) production to rate and frequency of $\mathrm{N}$ and $\mathrm{P} \mathrm{N}$ utrients Application at Areka, in southern Ethiopia.” Journal of Economics and Sustainable Development, Vol. 2 (7), pp. 423$431,2011$.

[3] E. Alehegn. "Maize Leaf Diseases Recognition and Classification Based on Imaging and Machine Learning Techniques." International Journal of Innovative Research in Computer and Communication Engineering, Vol. 5, 12, Dec 2017.

[4] G. Tigistu." Automatic Flower Disease Identification Using Image Processing." M. Sc. Thesis, Addis Ababa University, Addis Ababa, Ethiopia, 2015.

[5] A. Debasu et. al. "Ethiopian Coffee Plant Diseases Recognition Based on Imaging and Machine Learning Techniques." International Journal of Database Theory and Application, Vols. 9 (4), pp. 79-88, 2016.

[6] Quimio, A. and Mesfine T. "Diseases of enset." Proceedings of the International Work, 1996.

[7] M. G. Welde. "Variation in isolates of Enset wilt pathogen and reaction of enset clones to this disease." Addis Ababa, Ethiopia, App 2000.

[8] Luis Pérez-Vicente $\mathrm{PhD}$, Miguel A. Dita, $\mathrm{PhD}$ and Einar Martínez- de la Parte, MSc. "Technical Manual Prevention and diagnostic of Fusarium Wilt of banana caused by Fusarium oxysporum f. sp. cubense Tropical Race 4.” Food And Agriculture Organization of the United Nations. May 2014.

[9] S. Arivazhagan, R. Newlin Shebiah, S. Ananthi, S. Vishnu Varthini. "Detection of unhealthy region of plant leaves and classification of plant leaf diseases using texture feature." CIGR Journal, Vols. 15 (1), Mar 2013.

[10] Saxena, L., \& Armstrong, L. "A survey of image processing techniques for agriculture." Proceedings of Asian Federation for Information Technology in Agriculture, pp. 401-413, 2014.

[11] Prof. Patil Ashish, Patil Tanuja. Professor. "Survey on Detection and Classification of Plant Leaf Disease in Agriculture Environment." International Advanced Research Journal in Science, Engineering and Technology, National Conference on Innovative Applications and Research in Computer Science and Engineering (NCIARCSE-2017), Vol. 4, 4, Jan 2017.

[12] R. C. Gonzalez, R. E. Woods. Digital Image Processing. Addisen-Wesley, 1992.

[13] Kragujevac, J. Math. Image Preprocessing Tool, Olga Miljkovic, College of Computer Science, Megatrend University of Belgrade, 11000 Novi Beograd, Serbia. 2009.

[14] M. Sonka, V. Hlavac and R. Boyle. Image Processing. Analysis And Machine Vision. International Student Edition (3rd).

[15] P. Sivakumar (Research Scholar), Dr. S. Meenakshi. "A 
Review On Image Segmentation Techniques.” (IJARCET), Vol. 5, 3, Mar 2016.

[16] Sh. Medhi, Ch. Ahmed and R. Gayan. "A Study on Feature Extraction Techniques in Image Processing." International Journal of Computer Sciences and Engineering, Vol. 4, 7, Dec 2016.
[17] Y. Zhang and L. Wu, School of Information Science and Engineering, Southeast University, Nanjing, China. "An MRI Brain Images Classifier via Principal Component Analysis and Kernel Support Vector Machine." Progress in Electromagnetics Research, Vol. 130, pp. 369-388, 2012. 